\title{
Erratum: Vertex function compliant with the Ward identity for quasiparticle self-consistent calculations beyond $G W$ [Phys. Rev. B 103, L161104 (2021)]
}

\author{
Alexey Tal@, Wei Chen, and Alfredo Pasquarello \\ (Received 8 September 2021; published 25 October 2021)
}

DOI: 10.1103/PhysRevB.104.159901

The applied range-separated vertex has erroneously been described in the original paper. Equation (5) in the Supplemental Material of the paper should be replaced with the following description.

For $\mathbf{G}=\mathbf{G}^{\prime}$, the vertex is expressed as

$$
f_{\mathrm{xc}, \mathbf{G}, \mathbf{G}}^{\mathrm{LR}+\mathrm{LDA}}(\mathbf{q}, \omega)=\frac{1-Z(\mathbf{q}=0, \omega=0)}{\chi_{0}^{00}(\mathbf{q}, \omega=0)} e^{-|\mathbf{q}+\mathbf{G}|^{2} / k_{\mathrm{TF}}^{2}}+f_{\mathrm{xc}, \mathbf{G}, \mathbf{G}^{\prime}}^{\mathrm{LDA}}\left(1-e^{-|\mathbf{q}+\mathbf{G}|^{2} / k_{\mathrm{TF}}^{2}}\right),
$$

whereas for $\mathbf{G} \neq \mathbf{G}^{\prime}$ it reads

$$
f_{\mathrm{xc}, \mathbf{G}, \mathbf{G}^{\prime}}^{\mathrm{LR}+\mathrm{LDA}}(\mathbf{q}, \omega)=f_{\mathrm{xc}, \mathbf{G}, \mathbf{G}^{\prime}}^{\mathrm{LDA}}\left(1-\delta_{\mathbf{G}, 0}\right)\left(1-\delta_{0, \mathbf{G}^{\prime}}\right) .
$$

Hence, in the applied vertex, the off-diagonal terms of $f_{\mathrm{xc}}^{\mathrm{LDA}}$ are not included in the wings of the matrix. The results and the discussion in the original paper retain their validity.

Moreover, we found out that a further increase in the number of orbitals updated in the self-consistent iterations (NBANDSGW) beyond its default value leads to slightly improved converged values. These numerical differences do not affect any of the conclusions drawn in the original article. For reference, these updated results together with an improved extrapolation scheme are provided in Ref. [47] of the original paper.

[47] Materials Cloud Platform, 10.24435/materialscloud:hb-v3. 